\title{
18 Bodybuilding und Muskelaufbaupräparate
}

\author{
Melanie Richter und Karin Pöhlmann
}

\subsection{Kurze Darstellung des geschichtlichen und kulturellen Hintergrundes}

Schon in der Antike stellte ein athletischer, muskulöser Körper das Schönheitsideal des Mannes dar. Weit ab von der heutigen Bedeutung des Bodybuildings bezog sich das damalige Interesse am muskulösen Körper und an Muskelkraft jedoch eher auf die Entwicklung sportlicher Wettkämpfe und wehrhafter Heere. Dennoch galt schon zu dieser Zeit ein trainierter Körper als Zeichen für Lebens- und Geisteskultur (Müller 2011; Weinmann 2004). Dass Bodybuilding sich auf das klassische Schönheitsideal bezieht, zeigt sich in den „klassischen“ Posen von Eugen Sandow ebenso wie in der Namensgebung von Charles Atlas (eigentlich: Angelo Siciliano). Eine eindeutige Festlegung auf „den“ Begründer des Bodybuildings ist aufgrund einer Vielzahl von Personen, die die Entwicklung des Bodybuildings prägten, schwer. Die Wurzeln der Bodybuilding-Bewegung liegen besonders im europäischen Zirkus- und Varietémilieu des ausgehenden 19. Jahrhunderts (Müller 2011). Einer der wichtigsten Wegbereiter war Karl-Friedrich-Müller (1867-1925), besser bekannt als Eugen Sandow. Als Zirkusattraktion des US-amerikanischen Theaterproduzenten Florence Ziegfeld trat er als „der stärkste Mann der Welt" auf die Bühne und begeisterte das Publikum mit seinem außergewöhnlich muskulösen Körper und seinen kraftvollen Kunststücken. Seine spektakulären Aufführungen be- 
inhalteten beispielsweise das Stemmen von Pferden oder mehrerer Personen (Scheller 2010). Neben der Bewunderung der enormen körperlichen Stärke rückte durch Sandow erstmalig die körperliche Ästhetik des durchtrainierten Mannes in den Fokus der Aufmerksamkeit (Müller 2011; Schwarzenegger 1986). Am 14. September 1903 rief Sandow in London den ersten Bodybuilding-Wettkampf ins Leben, der unter dem Namen „Great Competition“ bekannt wurde. In seinem 1905 erschienen Buch „Bodybuilding or Man in the Making“ etablierte er als erster die Bezeichnung Bodybuilding (Scheller 2010).

In den letzten Jahren des 19. Jahrhunderts wurde durch die Schwerathleten, die in öffentlichen Vorstellungen ihre Kraft zeigten, das heutige Verständnis des Bodybuildings geprägt (Würzberg 1987). Sie trainierten in Vereinen für den Muskelaufbau und -zuwachs (Wedemeyer-Klowe 2004). Auch in der Sportart des Gewichthebens entwickelte sich ein zunehmendes Interesse am muskulösen Körper. Hier stand der Körper jedoch nicht vorrangig wegen seiner äußeren Erscheinung im Vordergrund. Das Kraftmessen und die Unterhaltung der Zuschauer prägten in dieser Zeit die Bedeutung des Sportes (Schwarzenegger 1986). Theodor Siebert (1866-1961) gründete 1901 in Deutschland erste Schulen für Athletik und Körperkultur und entwickelte auf der Basis physiologischer Kenntnisse erste Trainingsmethoden und Übungsabfolgen für das Bodybuilding (Wedemeyer 1999).

In den USA begann ab etwa 1930 eine neue Ära des Bodybuildings. Am Strand von Santa Monica schloss sich eine Gruppe von Männern zusammen, um ihre Körper in der Öffentlichkeit zu trainieren. Der sogenannte „Muscle Beach“ zog neben vielen Sportlern auch Zuschauer an, die neben der Kraft vor allem die ausgeprägte Muskulatur bestaunten und bewunderten. Auch die Brüder Ben und Joe Weider trainierten gelegentlich an diesem Strand. Joe Weider gründete 1939 die Zeitschrift „Your Physique“, die eine wichtige Informationsquelle für das Training bzw. Bodybuilding war. Er gründete 1946 in Montreal auch den ersten Bodybuilding-Verband, die „International Federation of Bodybuilding“(IFBB), und erreichte damit eine Trennung von Gewichtheben und Bodybuilding. Im Gegensatz zum Gewichtheben stehen im Bodybuilding die Kunst am Körper und die Ästhetik im Mittelpunkt (Müller 2011). Nachdem der Muscle Beach aufgrund von Beschwerden der Anwohner über die sexuelle Freizügigkeit von der Stadtverwaltung geschlossen wurde, entstanden nach und nach die ersten Fitnessstudios. Einige der bekanntesten Besitzer der ersten Studios waren beispielsweise Vic Tancy, Abby Pudgy Stockton oder Jack LaLanne. Zudem kamen immer mehr Zeitschriften auf den Markt, die das Wissen um das Bodybuilding rasant verbreiteten (,Muscle Training Illustrated“ Hrsg. Dan Lurie 1965; „Muscular Development“ Hrsg. John Grimek 1964). Auch in Deutschland kamen in den 1950er-Jahren eine Reihe von Bodybuilding-Magazinen auf, die Berichte über optimales Training, Ernährung und Lebensstil sowie Reportagen über Bodybuilding-Meisterschaften enthielten (Müller 2011). Auch erste Hanteln konnten käuflich erworben werden. Vince Gironda publi- 
zierte in den 1950ern erste Theorien zur Ernährungs- und Trainingsmethoden. Rheo H. Blair (geborener Irvin Johnson), ein junger Ernährungsexperte und Apotheker, forschte im Bereich der Bodybuilder-Ernährung und entwickelte in den 196oern die modernen Proteinsupplemente (das Rheo Blair Protein). Max Sick (1862-1961) entwickelte außerdem eine Trainingsmethode, welche allein aus Konzentrations- und Anspannungsübungen ohne Geräte bestand. Er gilt als Begründer des heutigen Posing-Bodybuilding (Spitz 1989). 1940 veranstaltete die AAU (Amateur Athletic Union) den ersten wirklichen Bodybuilding-Wettkampf unserer Zeit und verlieh dem Gewinner, den „Mister America“-Titel. 1947 wurde Steve Reeves „Mister America“. Er wurde durch seine Filmrollen in „Herkules“ und anderen sogenannten „Sandalenfilmen“ ein Vorbild für viele Männer (Müller 2011).

In Großbritannien wurde 1950 die National Amateur Body-Builders' Association (NABBA) gegründet, die Bodybuilding-Wettkämpfe veranstaltete. In den folgenden Jahren entstanden auch in Deutschland und anderen Ländern eigene NABBA Organisationen. Für professionelle Bodybuilder organisierte 1965 Joe Weider erstmals den Mister Olympia-Wettbewerb und führte eine endgültige Trennung von Krafttraining und Bodybuilding ein (von Stockert 1986). Arnold Schwarzenegger errang den Titel des „Mister Olympia“ insgesamt sieben Mal (Scheller 2012). Seit 1970 ist die IFBB als offizieller Bodybuilding-Weltverband Mitglied der General Assembly of International Sport (GAISF). Bis heute ist Bodybuilding allerdings keine olympische Disziplin.

\subsection{Ausprägungen und gängige Formen}

„Bodybuilding ist eine Form der Körpermodifikation“ lautet der erste Satz des deutschen Wikipedia-Eintrags zum Thema Bodybuilding (Wikipedia). Der Begriff Bodybuilding bezeichnet das Streben danach, die Muskelmasse durch Krafttraining an Fitnessgeräten zu erhöhen. Der Aufbau von Muskeln wird auch durch eine eiweißreiche Ernährung unterstützt. Bodybuilder streben danach, die Proportionen ihres Körpers zu verbessern und die maximale Muskelmasse und Muskeldefinition zu erreichen. Muskeln sind damit nicht Mittel, im Sinne von Muskelkraft wie etwa beim Gewichtheben, sondern das wesentliche Ziel der Aktivität, die einem ästhetischen Ideal von Masse, Symmetrie und Proportion folgt. Letztlich geht es nicht nur um eine Formung des Körpers, sondern um eine Umformung des Körpers. Aus dem, was die Natur einem gegeben hat, kann durch Mittel wie Training, Ernährung und Doping etwas Neues geschaffen werden. Natürliche Grenzen werden überwunden, der Körper wird zum „Werk“(s. Abb. 1). 

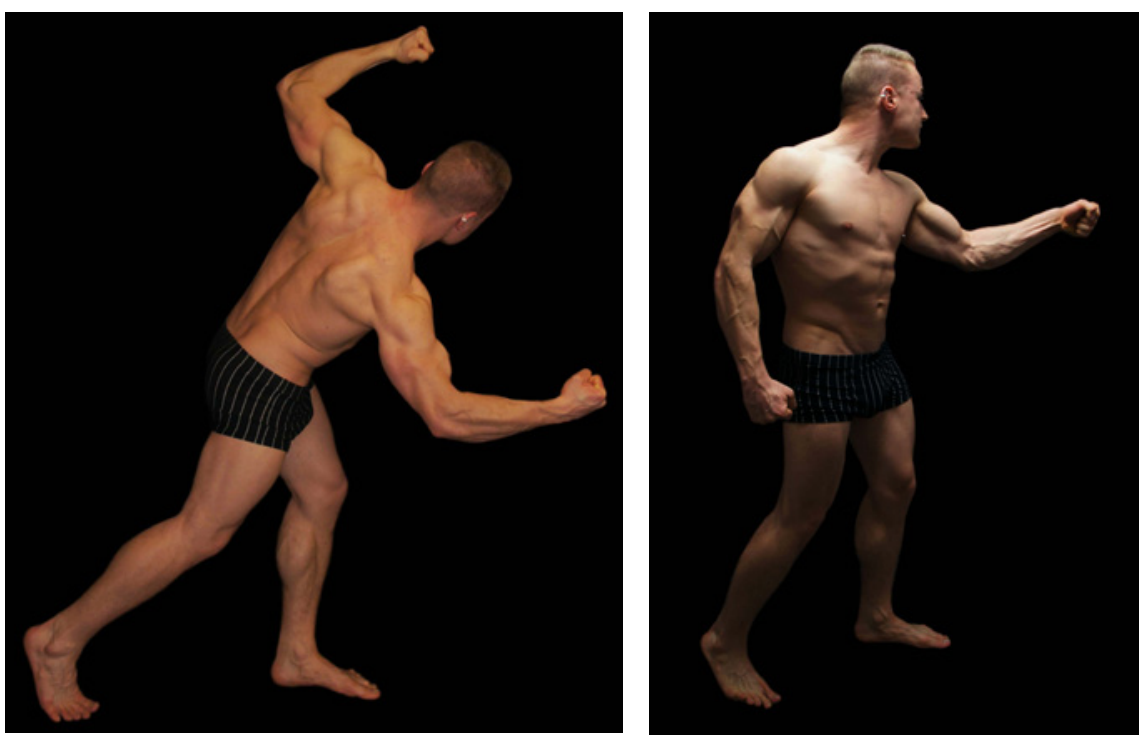

Abb. 1 Bodybuilding

\subsubsection{Muskelaufbau im Freizeitsport}

Die eigene Figur durch einen moderaten Aufbau von Muskeln und die Verbesserung des Muskeltonus zu verbessern ist für viele Fitnessstudio-Besucher ein Ziel. Für dieses „Body-Shaping“ gibt es neben Bodybuilding aber eine Vielzahl von Angeboten. Die Abgrenzung zum Bodybuilding lässt sich letztendlich nur durch die individuellen Zielsetzungen des einzelnen Trainierenden definieren. Bodybuilder streben danach, das Maximum an proportionaler Muskelmasse zu entwickeln.

\subsubsection{Bodybuilding als Wettkampfdisziplin}

In Abgrenzung zu dem Ziel, Muskeln aufzubauen, das viele Nutzer von Fitnessstudios haben, ist Bodybuilding zum einen durch die Art und Häufigkeit des Trainings definiert, zum anderen dadurch dass die Personen, die es ausüben, an Wettkämpfen teilnehmen oder teilnehmen möchten (z.B. Pickett et al. 2005; Parish et al. 2010). In professionellen Bodybuilding-Wettkämpfen werden jeweils mehrere Wertungsrunden durchgeführt, in denen die Hauptkriterien Muskelmasse, Proportion und Muskulosität sind. Die Einteilung der Teilnehmer erfolgt nach Alters- und Gewichtsklassen. In der ersten Runde wird die Reihe der Teilnehmer nach Symmetrie, Harmonie, Körperbau, Gleichmäßigkeit der Muskelentwicklung und Gesamteindruck bewertet. Anschließend werden jeweils mehrere Athleten hinsichtlich bestimmter Posen vergleichend bewertet. Bei Männern gibt es sieben Pflichtposen, Abbildungen 
der Pflichtposen finden sich z.B. auf der Webseite des Deutschen Bodybuilding und Fitnessverbands (www.dbfv.de). Im Finale werden die sechs besten Teilnehmer einer Klasse hinsichtlich der Ausführung der Pflichtposen vergleichend bewertet. Im sogenannten Posedown haben die Teilnehmer im Classic Bodybuilding 6o Sekunden Zeit, in denen sie beliebige Posen oder Elemente ihrer Kür zeigen können. Im Classic Bodybuilding gibt es außerdem eine dritte Runde, in der die Teilnehmer eine Kür zeigen, die 6o Sekunden dauert und musikalisch begleitet ist. In die Wertung der Kür fließen der Bewegungsablauf, die Übergänge zwischen einzelnen Posen, das Umsetzen der Musik in Bewegung unter Berücksichtigung von Proportion und Muskulosität sowie die kreative Gestaltung ein.

\subsubsection{Natural Bodybuilding}

Natural Bodybuilding bezeichnet die natürliche Form des Muskelaufbaus und Fettabbaus. „Natürlich“bedeutet dabei, dass keine illegalen Dopingsubstanzen, wie anabole Steroide, Diuretika und andere Drogen, die im WettkampfBodybuilding häufig sind, eingesetzt werden. Damit spiegelt das Natural Bodybuilding die Rückbesinnung auf die ursprünglichen Werte des Bodybuildings, nämlich die ästhetische Qualität mit ausgewogenen Muskelproportionen und nicht die reine Quantität im Sinne der höchstmöglichen Muskelmasse wider. Allein durch die drei „Eckpfeiler“ Training, Ernährung und Erholung soll das natürliche Potenzial an Muskelwachstum ausgeschöpft werden. Jedoch gibt es ein „genetisches Limit“, was bedeutet, dass ab einer individuell genetisch determinierten Muskel-Fett-Relation kein weiterer natürlicher Zuwachs an Muskelmasse möglich ist.

Das Natural Bodybuilding ist vom Muskelaufbau im Freizeitsport abzugrenzen. Es wird nach individuell aufgestellten Trainingsplänen bis zu fünf Mal in der Woche trainiert, wobei die Sportler an die Grenzen ihrer Leistungsfähigkeit gehen. Außerdem wird sehr streng auf eine ausgewogene, vor allem eiweißreiche Ernährung geachtet, bei der Nahrungsergänzungsmittel erlaubt sind (Breitenstein 2007). Auch im Natural Bodybuilding ist die Teilnahme an Wettkämpfen möglich, bei denen sich die Athleten freiwillig strengen Dopingund sogar Lügendetektortests unterziehen.

\subsection{Häufigkeiten}

\subsubsection{Bodybuilding}

Eine Aussage darüber, wie viele Menschen in Deutschland bzw. weltweit Bodybuilding betreiben, ist schwierig, da die Abgrenzung zwischen Bodybuilding und anderen „Body-Shaping“-Methoden nicht über die Aktivitäten per se, sondern letztlich nur über die Intention des Trainierenden möglich ist. 
Bisher gibt es keine Studien über die Häufigkeit des Bodybuildings in Deutschland oder anderen Ländern. Angaben zur Häufigkeit können indirekt aus anderen Indikatoren erschlossen werden. Ein Indikator ist die Anzahl der Fitnessstudios: 2002 gab es in Deutschland 6.500 Fitnessstudios mit 4.6 Millionen Mitgliedern (EMNID-Studie Fit-/Wellnessmarkt 2002), 2009 waren 6.31 Millionen Deutsche Mitglied in einem Fitnessclub (Deloitte/DSSV-Studie 2010), Ende 2011 gab es 7.300 Fitnessstudios mit 7.6 Millionen Mitgliedern (Deloitte/DSSVStudie 2011). Insgesamt sind damit 9,3\% der Deutschen in einem Fitnessstudio aktiv. In den Niederlanden und den USA sind es 15\% der Bevölkerung (Deloitte/ DSSV-Studie 2011).

Wie viele Mitglieder von Fitnessstudios Bodybuilding betreiben, kann nur geschätzt werden. Daten zur Rangfolge individueller Gründe für den Besuch eines Fitnessstudios, die im Auftrag des Arbeitgeberverbandes deutscher Fitness- und Gesundheitsanlagen (DSSV e.V.) 2008 erhoben wurden (DSSV e.V. 2008) zeigen, dass gesundheitliche Aspekte, wie die Steigerung der Fitness und die Erhöhung des Wohlbefindens, die primären Gründe für den Besuch von Fitnessstudios sind. An dritter Stelle der Rangreihe individueller Gründe steht das Motiv, das eigene Aussehen durch eine Gewichtsreduktion zu verbessern. Das Aussehen durch mehr Muskeln zu verbessern steht mit einem Anteil von 10\% erst an fünfter Stelle der individuell gewichteten Gründe für den Besuch eines Fitnessstudios. Nach Informationen des National Athletic Commitee Germany (NAC, Kau 2013) verfolgen etwa 300.000-400.000 Mitglieder bei ihrem Training ein Bodybuilding-Ideal, maximal 15.000 von ihnen haben schon einmal an Bodybuilding-Wettkämpfen teilgenommen.

Eine weitere Informationsquelle zur Verbreitung des Bodybuildings sind Mitgliedszahlen der Bodybuilding-Verbände. Aktuell gibt es in Deutschland sechs Bodybuilding- und Natural Bodybuilding-Verbände. Dazu zählen der Deutsche Bodybuilding- und Fitness-Verband e.V. (DBFV) und seine Landesverbände, das National Athletic Commitee Germany (NAC), die National Amateur Bodybuilding Association Germany (NABBA), die World Amateur Body Building Association Germany (WABBA) sowie die World Fitness Federation (WFF) und die German Natural Bodybuilding Fitness Federation (GMBF). Jeder dieser Verbände hat mehrere hundert Mitglieder. An Bodybuilding-Wettkämpfen haben laut den Ergebnislisten im Jahr 2012 etwa 600 Bodybuilder teilgenommen. Bei den deutschen Meisterschaften in Bochum im Dezember 2012 nahmen beispielsweise 127 Bodybuilder teil. An der Deutschen Natural Bodybuilding Meisterschaft nahmen 2012137 Athleten und Athletinnen teil.

\subsubsection{Muskelaufbaupräparate}

1998 ergab eine Befragung von 252 Mitgliedern aus 24 norddeutschen Fitnessstudios, dass $24 \%$ der männlichen und $8 \%$ der weiblichen Fitnessstudiobesucher regelmäßig anabole Steroide einnehmen (Boos et al. 1998). In einer deut- 
schen Multicenter-Studie (Boos et al. 2001) betrug der Anteil der Freizeitsportler, die anabole Steroide einnahmen, 19\% (22\% der Männer; 8\% der Frauen). Dabei ergab sich eine zweigipflige Altersverteilung von 20-25 Jahren und über 30 Jahren. Konsumierte Substanzen waren anabole Steroide (88\%), Stimulantien (22\%) und Wachstumshormone (2\%). Der überwiegende Anteil der Konsumenten $(48,1 \%)$ erhält die Substanzen durch das Gesundheitssystem (Melnik 2009).

Im deutschen Amateur-Bodybuilding wenden nach der Aussage des Arztes und Doping-Forschers Luitpold Kistler von 2007, etwa 30\% der Männer und 5\% der Frauen regelmäßig illegale Muskelaufbaupräparate an. Analysen von Urinproben im Zeitraum von 1995-2000 zeigten eine Missbrauchsrate von 39\% bei Amateur-Bodybuildern in Deutschland (Bundesinstitut für Sportwissenschaft 2001). Im professionellen Bodybuilding liegt Schätzungen zu Folge die Dopingrate bei 100\% (Kister 2007), da die dort gezeigten Ausmaße der Muskelausprägung durch natürlich Methoden nicht erreichbar sind. Insgesamt ist davon auszugehen, dass im Breiten- und Freizeitsport, in Fitness-Studios und in der Bodybuilding-Szene ca. 20-40\% der sportlich Aktiven Dopingmittel wie anabole-androgene Steroide und Stimulanzien einsetzen (Vogt 2007).

Der Bericht World Traffic in Doping Substances (Donati 2007) gibt an, dass jährlich weltweit 700 Tonnen anabole androgene Steroide (AAS) zu Dopingzwecken von 15 Millionen Konsumenten missbraucht werden. 38-40\% davon von Bodybuildern, Fitnessstudiokunden, Türstehern und Bodyguards. In den Vereinigten Staaten wurden im Jahr 2011 über 3 Millionen AAS- Konsumenten gezählt, wobei die Anzahl stetig zunimmt (De Souza u. Hallak 2011).

\subsection{Psychische Aspekte: Motive der Klienten}

\subsubsection{Männlichkeitsideal und Schönheitsideal}

Neben Steeve Reeves haben auch andere Bodybuilder, wie z.B. Lou Ferrigno oder Ralf Möller, den Übergang zu B-Movies geschafft. Auch Sean Connery war Bodybuilder, bevor er James Bond wurde. Der bekannteste Bodybuilder ist nach wie vor Arnold Schwarzenegger, der zwischen 1970 und 1980 sieben Mal den Titel „Mister Olympia“ errang, für den Bodybuilding die Grundlage seines sozialen Aufstiegs zum Hollywood Filmstar und schließlich zum Politiker war. Er verkörperte zusammen mit Sylvester Stallone das Körperideal der 8oer-Jahre. Ebenso wie das physische Merkmal Schlankheit mit psychischen Eigenschaften wie Selbstkontrolle und Leistungsfähigkeit verknüpft ist (Brownell 1991), wird auch eine Verbindung zwischen dem harten, muskulösen Männerkörper und mentaler Stärke hergestellt (z.B. Jefferson 1998). Muskeln sind damit vom Unterschichtsmerkmal zum Statussymbol geworden. Dass ein durchtrainierter und muskelbepackter Körper mit dem Sixpack als Statussymbol zum allgemein akzeptierten Schönheitsideal geworden war, zeigte Anfang 
der goer-Jahre auch die Calvin Klein-Werbekampagne für Unterwäsche, in der Marky Mark und Kate Moss männliche und weibliche Schönheitsideal verkörperten: „buff“-Look für Männer, „waif“-Look für Frauen.

Bei Männern hat sich das Körperideal in den letzten Jahrzehnten in Richtung zunehmender Körpergröße und Muskelmasse bei gleicher Taillenweite verändert, dieser Trend lässt sich z.B. anhand von Actionfiguren aus den letzten 25 Jahren zeigen (Baghurst et al. 2006). Auch in der Darstellung von Männerkörpern in Magazinen wie GQ, Sports Illustrated und Rolling Stone wurden von 1967 bis 1997 zunehmend schlankere und muskulösere Männerkörper mit einer ausgeprägten V-Form (breite Schultern, schmale Taille) dargestellt. Schlankheit und die V-Form des Oberkörpers nahmen bis zu den 8oer-Jahren deutlich zu und danach wieder leicht ab. Bis zu den goern wurden zunehmend muskulösere Männer dargestellt (Law u. Labre 2002). Auch für Männerkörper, die von 1973 bis 1997 im Magazin Playgirl dargestellt wurden, zeigte sich der Trend, dass die Muskelmasse zunimmt und der Körperfettanteil abnimmt (Leit et al. 2001). Auch in Fernseh-Werbespots werden am häufigsten muskulöse Männer gezeigt. In 505 untersuchten Werbespots hatten 30,4\% der gezeigten Männer einen muskulösen Körper, dagegen waren nur 4,4\% bzw. 8,7\% unter- bzw. übergewichtig (Lin 1998).

Ebenso wie bei Frauen nimmt bei Männern die Diskrepanz zwischen in den Medien propagierten Körperidealen und dem eigenen Körper stetig zu. Diese Diskrepanz löst auch bei Männern Unzufriedenheit mit dem eigenen Körper aus. Sie sehen ihren eigenen Körper zunehmend kritisch und wünschen sich mehr Muskelmasse und Muskeltonus (Pope et al. 200o). Bevölkerungsrepräsentative Untersuchungen in den USA (Berscheid et al. 1973; Cash et al. 1986; Garner 1997) zeigen, dass der Anteil der Männer, die mit ihrem Körper unzufrieden sind, in den letzten Jahrzehnten erheblich zugenommen hat, er lag 1972 bei $15 \%$ und 1996 bei $43 \%$. Die Daten dieser Untersuchungen zeigen aber auch, dass die Unzufriedenheit nicht für alle Körperbereiche im gleichen Maß gestiegen ist. Der stärkste Anstieg der Unzufriedenheit bezog sich auf die Aspekte Taille - von $36 \%$ auf $63 \%$ - und Muskeltonus - von $25 \%$ auf $45 \%$. Auch die Bewertungen des Oberkörpers und des Gewichts veränderten sich innerhalb dieser 25 Jahre stark. Mit ihrem Oberkörper waren 1972 18\% der befragten Männer unzufrieden, 1996 waren es 38\%, für das Merkmal Gewicht zeigte sich ein Anstieg von 35\% auf 52\%. Zum Vergleich: Die Zufriedenheit mit der eigenen Körpergröße war in den 25 Jahren, die die drei Repräsentativbefragungen abdecken, relativ stabil: 1972 waren 13\% der Männer mit ihrer Körpergröße unzufrieden, 1996 waren es $16 \%$. Neuere Untersuchungen sprechen dafür, dass die Unzufriedenheit mit dem Körper bei Frauen und Männern seit Mitte der goer-Jahre nicht mehr weiter steigt, sondern stabil bleibt (Heatherton et al. 1995; Cash et al. 2004). Eine Erklärung für diese Befunde ist, dass andere Faktoren die Wirkung der medialen Körperideale abmildern (Cash et al. 2004). Zu diesen Faktoren gehören z.B. die zunehmende Bewusstheit über Risikofakto- 
ren von Essstörungen und die reale Zunahme der Häufigkeit von Adipositas, die Abwärtsvergleiche fördert. Wenn die in den Medien repräsentierten Körperideale allerdings internalisiert werden und negative soziale Vergleichsprozesse mit dem realen eigenen Körper auslösen, kann dies eine Körperbildstörung auslösen, die auf das Bestreben fokussiert ist, den eigenen Körper muskulöser zu machen (drive for muscularity, z.B. Daniel u. Bridges 2010).

\subsubsection{Motive für Bodybuilding}

Im Vergleich zu anderen Formen der Körpermodifikation gibt es nur wenige Studien, die untersuchen, warum Bodybuilder Bodybuilding als Weg gewählt haben, ihren Körper zu gestalten. In ihrer Untersuchung zu den Motiven von Bodybuildern benennen Parish et al. (2010) folgende Gründe, die in der Literatur beschrieben werden: Selbstwert, Nachahmung, Bodybuilding als Ersatz für andere Sportarten sowie Gesundheit.

Die Stabilisierung des Selbstwertes durch Bodybuilding ist nach der Einschätzung von Parish et al. (2010) der am häufigsten untersuchte Grund. Bestehende Selbstwertdefizite, die sich auf die eigene körperliche Erscheinung oder auf andere bei sich selbst wahrgenommenen psychischen Unzulänglichkeiten beziehen können, können durch Bodybuilding kompensiert werden (z.B. Fussell 1991; Klein 2007). Gefühle von Schwäche und Hilflosigkeit können hinter der physischen Fassade von Kraft und Stärke verborgen werden. Genauso wie Muskeln aufgebaut werden, kann der Selbstwert gesteigert und damit das Selbstbild stabilisiert werden. Diese lineare Beziehung gilt besonders für die körperbezogene Komponente des Selbstwerts. Je geringer der Körperselbstwert, desto intensiver wird trainiert (Olivardia et al. 2004; McCreary et al. 2007).

Ein weiterer Crund dafür, Bodybuilding zu betreiben, ist das Streben nach dem idealen männlichen Körper (Klein 2007), das von den Medien vorgegeben wird (s. Kap. 18.4.1). Bodybuilding kann außerdem ein Ersatz für andere Sportarten sein, die die Person früher intensiv betrieben hat. Bodybuilding ermöglicht dann, den Leistungs- und Wettbewerbsaspekt weiter auszuleben (z.B. Li 2004). Bodybuilding als Weg zu mehr Gesundheit wird sowohl von Bodybuildern als auch von den Bodybuilding-Verbänden propagiert. Die im Bodybuilding notwendige Ernährung wird für gesund gehalten, auch wenn sie einseitig ist (z.B. Perine 2008 zitiert nach Parish et al. 2010). Besser belegt sind positive Auswirkungen von Bodybuilding auf die psychische Gesundheit, vor allem auf Angst und Depression (z.B. Hurst et al. 200o; Zhang et al. 2002). In ihrer eigenen Untersuchung fanden Parish et al. (2010), dass das Streben danach, anderen realen Personen oder Idolen nachzueifern, mit 34\% der von den Befragten am häufigsten genannte Grund dafür war, Bodybuilding zu machen. Der zweithäufigste Grund war Bodybuilding als Ersatz für andere Wettkampfsportarten (31\%). Selbstwertbezogene Antworten standen erst an dritter Stelle 
der genannten Gründe für Bodybuilding (14\%), Gesundheit wurde von jedem zehnten der Befragten als Grund genannt. Die Ergebnisse dieser Studie unterstreichen die hohe Bedeutung des Schönheits- und Männlichkeitsideals für Bodybuilder.

\subsubsection{Bodybuilding als hierarchischer Selbstdefinitionsprozess}

In seiner Bedeutung als Ausdrucksmittel für das Selbst der Person wird der Körper heute als Element der Identität verstanden, das man gestalten kann und muss (z.B. Pöhlmann u. Joraschky 2006). Die Gestaltung des Körpers durch Bodybuilding stellt eine hierarchische Selbstdefinition (Baumeister 1986) dar, die durch quantitative Indikatoren wie Muskelmasse und Muskeldefinition bestimmt werden kann. Auch andere im Bereich der körperlichen Erscheinung angestrebte Eigenschaften wie Schlankheit, Fitness oder Attraktivität lassen sich als hierarchische Selbstdefinitionsprozesse verstehen. Beispiele für hierarchisch definierte Identitätskomponenten außerhalb des Körpers sind Bildung oder Reichtum. Für Identitätskomponenten, die sich hierarchisch durch ein „mehr“ oder „weniger“ von etwas definieren lassen, gibt es keine Kriterien, die bestimmen, wann man sie erreicht hat. Der Prozess des Erwerbs von Identitätskomponenten auf der Basis von hierarchischen Kriterien ist nie abgeschlossen und muss immer neu definiert werden. Selbstdefinitionen dieses Typs bringen außerdem den Vergleichs- und Wettbewerbsaspekt in die Identität ein. Weil es relativ klar definierte, in diesem Fall quantitative Standards gibt, kann man sich mit anderen vergleichen und von ihnen abgrenzen. Quantitative Standards gelten im Fall von Bodybuilding nicht nur für das Ergebnis der Aktivität, das sich durch Muskelmasse, Muskeldefinition, Umfang einzelner Körperteile, Körperfettindex und Kriterien wie Symmetrie, Proportion und Ästhetik beschreiben lässt. Auch der Weg zum perfekt geformten Körper beinhaltet quantitative Indikatoren wie z.B. Anzahl der Sätze pro Übung, Anzahl der Wiederholung pro Satz, Höhe der Gewichte im Krafttraining.

Auch der Aspekt des sozialen Vergleichs trifft auf Körperbild-Komponenten wie Attraktivität, Fitness, Schlankheit, Muskelmasse oder Muskeltonus zu. In Berichten von Bodybuildern wird der Aspekt des sich Messens mit anderen Bodybuildern betont. Diese sozialen Vergleiche können ein Gefühl von Stolz und Gruppenzugehörigkeit (Giraldi 2011; Smith u. Stewart 2012) auslösen aber auch Scham und Minderwertigkeitsgefühle (Baghurst 2012; Mosley 2009).

Hierarchisch definierte Prozesse der Selbstdefinition sind außerdem nie abgeschlossen, sondern erfordern Aktivität und kontinuierliches Engagement der Person. Zusätzlich gilt für Selbstdefinitionen, die auf physischen Attributen beruhen, dass der Körper eine grundsätzlich instabile Identitätskomponente ist (Baumeister 1986). Während Reichtum und Bildung, die man sich erarbeitet hat, relativ stabil bleiben, unterliegt der Körper immer biologischen 
Veränderungen. Die durch Training, Ernährung und andere Aktivitäten des Bodybuilders erreichten Erfolge bleiben nicht stabil, sondern verschwinden wieder, wenn die notwendigen Aktivitäten nicht weitergeführt werden. Auch wenn die Wege zum Erreichen dieser Identitätskomponenten grundsätzlich transparent sind (Training, Ernährung, Doping), kann die Orientierung an einer nicht realisierbaren Zielvorstellung negative Konsequenzen für die Selbstregulationsprozesse der Person haben.

Auf dem Weg zum perfekten Körper müssen nicht nur körperliche Schmerzen ertragen und physische Grenzen überwunden werden, es geht auch um das Meistern der eigenen Emotionen und damit um die Entwicklung der Persönlichkeit. Zusammen mit dem bestmöglichen Körper entsteht damit auch das bestmögliche Selbst, postuliert z.B. LL Cool J (2011), der sich vom Moppel zum Muskelprotz transformiert hat. Auch in anderen Berichten wird beschrieben, dass der Auslöser, Bodybuilding zu betreiben, das Bedürfnis ist, eigene Unzulänglichkeiten zu überwinden, die subjektiv hinsichtlich Körpergröße oder Körpergewicht bestehen (Giraldi 2011; Breitenstein 2003).

Der Auslöser, Bodybuilding zu betreiben, kann ein negatives Körperbild sein. Das Körperbild ist ein Bestandteil des Selbstkonzeptes und beinhaltet Einstellungen, Gedanken, Gefühle und Verhaltensweisen, die den eigenen Körper betreffen (Cash 2004). Wenn die Körperakzeptanz, als kognitive Komponente des Körperbildes, gering ist, und der Selbstwert der Person dadurch beeinträchtigt ist oder dysfunktionale Verhaltensweisen zur Reduzierung der Diskrepanz zwischen Körperideal und Realkörper eingesetzt werden, liegt eine Körperbildstörung vor. Muskeln zu trainieren, ist dann ein Weg, körperliche Unzulänglichkeiten zu überwinden und die eigene Persönlichkeit zu verändern. Wie bei anderen Körpermodifikationen auch erlebt die Person Kontrolle über ihren Körper und erfährt Wirksamkeit bei seiner Gestaltung. Bodybuilding bietet im Gegensatz zu anderen Körpermodifikationen besonders viele quantitative Indikatoren, die diesen Transformationsprozess für die Person sichtbar und sogar messbar machen.

Die durch Bodybuilding geschaffenen Körper sind übermenschlich und übermännlich (Giraldi 2011; Smith u. Stewart 2012). Innerhalb der BodybuildingKultur wird dieser Leistungs- und Meisterungsaspekt exzessiv ausgelebt und von den Beteiligten geteilt. Smith und Stewart (2012) sprechen von einer Meritokratie der Muskeln, in der die Starken respektiert werden und die Riesen dominieren. Außerhalb der Subkultur werden die durch Bodybuilding geschaffenen Körperkunstwerke allerdings nicht nur bewundert. Auch der Deutsche Bodybuilding und Fitness Verband (www.dbfv.de) weist darauf hin, dass das Ziel von Bodybuilding auch der fitte, moderat muskulöse Körper sein kann und betont den Ausnahmecharakter der „monströsen“ Körper, die in Bodybuilding-Magazinen und Bodybuilding-Meisterschaften zur Schau gestellt werden. 


\subsubsection{Muskeldysmorphie}

Wenn die subjektive Bewertung der eigenen Muskelmasse deutlich von der Realität abweicht, kann eine psychische Störung Grund dafür sein. Das Phänomen, dass extrem muskulöse Bodybuilder sich selbst als schmächtig und wenig muskulös wahrnehmen, wurde von Pope et al. (1993) zum ersten Mal beschrieben und als „reverse anorexia“ bezeichnet, weil er in seiner Stichprobe von Bodybuildern eine hohe Prävalenz von früheren Essstörungen fand; auch die Bezeichnung Bigorexia beschreibt die inhaltliche Nähe zu Essstörungen. Später verwendeten Pope et al. (1997) allerdings die Bezeichnung Muskeldysmorphie (muscle dysmorphia), weil die Fehlwahrnehmung der Ausprägung der eigenen Muskelmasse als zentrales Kennzeichen der Störung aufgefasst wurde. Es handelt sich damit wie bei der Anorexie um eine Körperschemastörung, d.h. der Betroffene nimmt das dreidimensionale Raumbild seines Körpers (Schilder 1923) verzerrt wahr.

\section{Kennzeichen der Muskeldysmorphie}

Die Diagnose einer Muskeldysmorphie kann gestellt werden, wenn folgende Kriterien erfüllt sind (Mosley 2009; Murray et al. 2010):

- Der Betroffene ist überzeugt, dass der eigene Körper nicht muskulös und schlank genug ist. Diese Überzeugung führt zu extremen Trainingsverhalten und übermäßiger Fokussierung auf die Ernährung.

- Diese Sorge führt dazu, (a) dass soziale, berufliche oder Freizeitaktivitäten aufgegeben werden, weil der Betroffene zwanghaft bemüht ist, Trainings- und Diätpläne einzuhalten; (b) der Betroffene Situationen vermeidet, in denen andere seinen Körper sehen, bzw. derartige Situationen bei ihm Unruhe oder extreme Angst auslösen; (c) Die Beschäftigung mit wahrgenommenen Defiziten in Körpergröße und Muskelmasse führt beim Betroffenen zu deutlichen Belastungen oder Behinderungen in sozialen Beziehungen, im beruflichen Umfeld oder in anderen wichtigen Lebensbereichen; (d) Der Betroffene trainiert weiter oder wendet weiter Muskelaufbaupräparate an, obwohl er negative körperliche oder psychische Auswirkungen kennt. Zwei dieser vier Kriterien müssen erfüllt sein.

- Das Charakteristikum der Störung ist, dass die Überzeugung zu wenig Muskeln zu haben im Mittelpunkt steht und das Verhalten der Betroffenen darauf fokussiert ist.

- Differenzialdiagnostisch davon abzugrenzen ist die Überzeugung zu dick zu sein oder eine Fokussierung auf andere Aspekte der körperlichen Erscheinung im Sinne einer körperdysmorphen Störung.

Bodybuilder erreichen, im Vergleich mit anderen Sportlern, in deren Disziplin Muskeln wichtig sind, wie z.B. Gewichtheben und College Football, die höchs- 
18.5 Medizinische Aspekte: rechtliche Situation bzw. gesetzliche Regelungen, Nebenwirkungen, Wechselwirkungen

ten Werte für Muskeldysmorphie. Die Werte für Muskeldysmorphobie sind bei Bodybuildern, die Natural Bodybuilding ausüben, genauso hoch wie bei anderen Bodybuildern (Baghurst u. Lirgg 2009). Schätzungen gehen davon aus, dass etwa 10\% der Bodybuilder an Muskeldysmorphie leiden (Pope et al. 200o). Muskeldysmorphie führt nicht nur zu extremem Trainings- und Essverhalten, sie ist außerdem mit einem erhöhten Risiko für AAS-Missbrauch, Essstörungen (Babusa u.Túry 2012; Goldfield et al. 2006) sowie Angst- und affektiven Störungen (Cafri et al. 2008) verbunden.

\subsection{Medizinische Aspekte: rechtliche Situation bzw. gesetzliche Regelungen, Nebenwirkungen, Wechselwirkungen}

\subsubsection{Rechtliche Situation bzw. gesetzliche Regelung}

Rechtlich relevant ist im Kontext des Bodybuildings der Gebrauch von anabolen Steroiden. Im Jahre 1967 wurden nach einem Doping-Skandal bei der Tour de France von internationalen Sportverbänden wie dem Internationalen Radsportverband (UCI) und dem Internationalen olympischen Komitee (IOC) erstmals Anti-Doping-Regeln für den sportlichen Wettkampf formuliert (Clasing 2004; Schänzer u. Thevis 2007). Im Jahre 2004 übernahm die Welt-Anti-DopingAgentur (WADA) den Kampf gegen Doping im Sport (Schänzer u. Thevis 2007). Nachdem verbesserte Nachweisverfahren für die Abbauprodukte synthetischer Hormone entwickelt wurden, kam es erstmals zu einem Verbot von anabolen Substanzen. Seit 1984 ist auch die Anwendung des körpereigenen Steroidhormons Testosteron verboten (Clasing 2004). Jährlich veröffentlicht WADA eine aktuelle Verbotsliste (Haas 2004; WADA 2013) In Deutschland können anabole Steroide durch eine ärztliche Verordnung legal erworben werden. Sie werden beispielsweise bei Muskelschwund, Blutarmut, Pubertätsverzögerung oder bei älteren Männern, deren Fähigkeit Testosteron zu bilden stark reduziert ist, verschrieben (Bahsin 2000; Johnson 2000). Ohne Rezept ist der Erwerb von anabolen Steroiden in vielen Ländern, wie England, den USA und Australien verboten. Auch in Deutschland unterliegen anabole Steroide dem Arzneimittelschutzgesetz (Lippert 1999). In anderen Ländern hingegen, wie Mexico, Thailand oder Spanien, sind anabole Steroide auch ohne ärztliche Verordnung erhältlich.

Zur Verbesserung der Bekämpfung des Dopings im Sport, wurde in Deutschland im Jahre 2007 eine weitere Verordnung auf der Grundlage des $₫ 6 a$ Abs. 2a Satz 2 des Arzneimittelgesetzes eingeführt (Bundesregierung 30.03.2007; Rüdiger u. Parzeller 2007). Seither unterliegt der Besitz bestimmter zum Doping geeigneter und besonders gefährlicher Stoffe einem Besitzverbot, sofern es sich dabei um eine nicht geringe Menge handelt. Im Anhang des Gesetzes ist die zulässige Menge für die Dopingstoffe, die dem Besitzverbot unterliegen, aufgeführt (BGBI. 1994 S. 334). Mit Freiheitsstrafe bis zu drei Jahren oder mit Geldstrafe wird also nicht nur bestraft, wer ein verschreibungspflichtiges Arz- 
neimittel in den Verkehr bringt, bei anderen anwendet oder herstellt, sondern auch wer es in einer nicht geringen Menge besitzt. In besonders schweren Fällen kann eine Freiheitsstrafe von bis zu zehn Jahren ausgesprochen werden. Mit diesem Besitzverbot sollen bereits Vorstufen des Handels erfasst und damit der Weitergabe von Dopingmitteln sowohl im Spitzensport als auch im Breitensport wirksam entgegengewirkt werden.

\subsubsection{Medizinische Aspekte: Nebenwirkungen, Wechselwirkungen Nebenwirkungen des Maximalkrafttrainings}

Verletzungen durch Bodybuilding sind relativ selten und entstehen zum größten Teil beim Training (Steinbrück 1999). Hauptsächlich treten Muskel-, Gelenk- und Sehnenverletzungen durch Fehl- oder Überbelastung auf (Ritsch 2005). Dabei sind die oberen Extremitäten besonders häufig von Verletzungen betroffen. Bei männlichen Bodybuildern sind meist Schultern und Ellenbogen von Überlastungssyndromen, bei Frauen die Knie betroffen (Klein et al. 1979; Goertzen et al. 1989). Im Bereich der Supraspinatussehne, die bei Bodybuildern im Vergleich zur Normalbevölkerung oft um 50\% kräftiger ausgebildet ist (Jerosch et al. 1989), kommt es dabei am häufigsten zu Schmerzen und Entzündungen (Ritsch 2005). Ebenfalls häufig sind Sehnenansatzerkrankungen der hinteren Anteile der Rotatorenmanschetten (Musculus infraspinatus, Musculus teres minor) (Barlow et al. 2002). Im schlimmsten Fall entwickelt sich aus einer chronischen Supraspinatussehnenreizung ein Rotatorenmanschettenriss.

Auch im Bereich des Schultereckgelenkes (Akromioklavikulargelenk) sind sehr häufig überlastungsbedingte Reizungen zu verzeichnen (Ritsch 2005). Bei chronischer Überlastung dieses Gelenkes kann es zu einer frühzeitigen Arthrose oder gar einer Knochenauflösung (Osteolyse) des seitlichen Schlüsselbeinendes kommen (Ritsch 2005). In fortgeschrittenen Fällen muss das seitliche Schlüsselbeinende operativ entfernt werden.

Am Ellenbogengelenk kann es zu Insertionstendinopathien (Sehnenansatzerkrankungen) kommen (Ritsch 2005). Dazu gehört der Tennisarm (Epicondylitis humeri radialis). In gleicher Weise gibt es die Erkrankung am Ansatz der Unterarmbeugemuskulatur am Epicondylus humeri ulnaris (Golferarm). Sehnenoder Muskelrisse sind relativ seltene Ereignisse und beruhen auf einer degenerativen Vorschädigung der Sehne oder einer extremen Überlastung des Muskels. Im Bereich der distalen Bizeps- und Trizepssehne kommen die meisten Verletzungen vor (Ritsch 2005). Bei einem Riss besteht die Indikation zur operativen Befestigung der Sehne (Refixation). Im Handgelenksbereich überwiegen die Sehnenscheidentzündung (Tendovaginitis) der Beuge- oder Streckmuskulatur sowie Überlastungssymptome des Handgelenkes (Beuker et al. 1966).

Bei Verletzungen der unteren Extremitäten ist meist die Adduktorenmuskulatur, die vordere und hintere Oberschenkelmuskulatur betroffen (Bak et al. 
18.5 Medizinische Aspekte: rechtliche Situation bzw. gesetzliche Regelungen, Nebenwirkungen, Wechselwirkungen

200o; Shepsis et al. 2000). Auch Ansatztendinosen am Beckenring können durch diese Muskeln verursacht werden. (Beuker et al. 1966). Zu akuten Beschwerden kann es ebenfalls durch eine Blockierung des Iliosakrargelenkes (ISG) oder einer Fehlstellung des Kreuzbeines (Sacrum) kommen. Ansonsten treten auch im Bereich des Kniegelenkes Ansatztendinosen auf. Betroffen sind häufig die Quadrizepssehne und die Patellarsehne (Ritsch 2005).

Verletzungen der Wirbelsäule und Bandscheiben sind beim Bodybuilding eher seltene Ereignisse (Brown u. Kimball 1983; Granhead u. Morelli 1988).

\section{Nebenwirkungen durch anabole Steroide}

Anabole androgene Steroide (AAS) sind Steroide, zu denen das körpereigene Hormon Testosteron und seine Derivate gehören (Pope u. Brower 2005; Bahrke u. Yesalis 2004). Das Hormon Testosteron unterstützt im Körper den Eiweißaufbau durch die Förderung der Proteinsynthese und die Reduzierung eiweißkataboler Prozesse (anabole Wirkung). Außerdem sorgt es für die Ausbildung der sekundären und primären Geschlechtsmerkmale (androgene Wirkung) (Kochakian 1993). Im Training sinkt der Testosteronspiegel und Glucocorticoide bauen das Muskelgewebe ab. Synthetisch hergestellte AAS füllen das Testosteronlevel wieder auf und blockieren damit die muskelabbauenden Effekte des Glucocorticoides. Das heißt die anabole Wirkung wird verstärkt und die androgenen Effekte minimiert (Sheffield-Moore u. Urban 2004). Im Bodybuilding wird jedoch häufig das Hundertfache (1ooomg/Woche) der natürlichen physiologischen Dosis an AAS eingenommen (Parkinson u. Evans 2006). Welche langfristigen gesundheitlichen Gefahren damit einhergehen, ist bisher schwer zu erfassen, da ein eindeutiger Kausalschluss aufgrund einer Vielzahl von weiteren Einflussfaktoren, wie z.B. die kombinierte Einnahme von Medikamenten, die Wirkung der AAS infrage stellt. Eine Reihe von Untersuchungen geben jedoch Hinweise auf äußerliche kurzzeitige Nebenwirkungen sowie lebensbedrohliche Schädigungen des Herz-Kreislaufsystems, der Leber und Nieren.

\section{Herz}

Bei Bodybuildern, die Anabolika konsumierten, konnte in mehreren Studien eine deutliche linksventrikuläre Hypertrophie nachgewiesen werden (Vanberg u. Atar 2010; Yeater et al. 1996; Sachtleben et al. 1993). Zudem zeigte sich, dass der linke Ventrikel bei Anabolikakonsumenten deutlich dicker war als in einer Vergleichsgruppe von Bodybuildern, die keine anabolen Steroide einnahmen (McKillop et al. 1986; Payne et al. 2004). Auch Myokardfibrosen und Myokardnekrosen wurden nach chronischem Missbrauch von AAS beschrieben (Vanberg u. Atar 2010; Sullivan et al. 1998). 


\section{Vaskuläres System}

Durch die chronische Einnahme von AAS kommt es zu einer Veränderung der Blutfettwerte. Dabei zeigt sich eine Abnahme des vor Gefäßverkalkung schützenden High Density Lipoproteins (HDL)-Spiegels (Hartgens et al. 2004; Vanberg u. Atar 2010) und eine Zunahme des Low Density Lipoproteins (LDL) (Dickerman 1997). Die Folge können vermehrte Ablagerungen an den Arterienwänden sein, die das Risiko einer koronaren Herzerkrankung erhöhen (McCarthy et al. 2000; Nieminen et al. 1996; Hurley et al. 1984).

Inwieweit anabole Steroide ursächlich für Schlaganfälle sein können, ist bisher noch nicht eindeutig nachzuweisen. Einzelne Fallberichte beschreiben jedoch hämorrhagische und ischämische Schlaganfälle nach der Einnahme von AAS (Lisiewicz et al. 1999; Frankle et al. 1988).

\section{Leber}

Die Einnahme von AAS kann zu Schädigungen der Leberzellen (Stimac et al. 2002), Cholestase (Velayudham u. Farrell 2003), blutgefüllten Zysten (Peliosis hepatis) (Cabasso 1994), Adenomen (Nakao et al. 200o) bis hin zu einem hepatozellulären Karzinom (Martin et al. 2008) und Leberversagen (Ryan 1981) führen.

\section{Nieren}

Einzelne Fallberichte beschreiben entzündliche Nierenkrankheiten (Hartung et al. 2001; Revai et al. 2003), wobei die direkte oder indirekte Wirkung der AAS noch ungeklärt ist. Zudem werden in einigen Fällen akutes Nierenversagen und Nierenzellkarzinome im ursächlichen Zusammenhang mit der AAS Einnahme vermutet (Habscheid et al. 1999; Martorana et al. 1999).

\section{Haut}

Auch im dermatologischen Bereich treten Nebenwirkungen durch die Einnahme von anabolen Steroiden auf. Dabei führt der AAS-Konsum zu einer Hypertrophie der Talkdrüsen. Die vermehrte Talksekretion verursacht besonders im Cesichts-, Brust- und Rückenbereich fettige Haut und unterschiedlich stark ausgeprägte Akne, wobei das Spektrum von geringgradiger Acne vulgaris bis zu Acne conglobata oder Acne fulminans reicht (Melnik et al. 2007; Mayerhausen u. Riebel 1989; Kraus et al. 2012). Bedingt durch mit der Einnahme einhergehende Leberschäden kann es auch zu einer Gelbfärbung der Haut kommen. Zudem sind Überdehnungsnarben im Bereich der oberen Extremitäten häufig (Scott et al. 1994).

\section{Spezielle Nebenwirkungen bei Männern}

Die Einnahme anaboler Steroide kann bei Männern die Freisetzung des Gonadotropin releasing hormone $(\mathrm{GnRH})$ und damit die Gonadotropinproduktion 
18.5 Medizinische Aspekte: rechtliche Situation bzw. gesetzliche Regelungen, Nebenwirkungen, Wechselwirkungen

hemmen. Folglich vermindert sich die Blutkonzentration des follikelstimulierende Hormons (FSH) und des luteinisierende Hormons (LH) und es kommt zu einem Anstieg des Östradiols und einer Unterdrückung der Testosteronproduktion. (Birkeland et al. 1994; Bhasin et al. 2001). Dieser suppressive Effekt bewirkt bei Männern eine Feminisierung. Dabei ist vor allem die Produktion der Spermazellen betroffen. Die Morphologie der Spermatozoen ist deutlich verändert und die Spermienmotilität reduziert (Torres-Calleja et al. 20oo, 2001). Weitere Nebenwirkungen sind eine Verkleinerung der Hoden (Hypogonadismus) (Birkeland et al. 1994; Bain 2007) und ein Wachstum der Brust (Gynäkomastie) (Neild 1995). In einzelnen Fallberichten wird das Auftreten von malignen Hoden- bzw. Prostatatumoren (Froehner et al. 1999) in Zusammenhang mit der Einnahme von AAS beschrieben.

\section{Spezielle Nebenwirkungen bei Frauen}

Die durch den AAS-Konsum verursachte Hemmung des FSH und LH, die mit einer verminderten Produktion an Östrogen und Progesteron in den Ovarien verbunden ist, bewirkt bei Frauen eine Virilisierung. Dazu zählen die Hypotrophie der Brust, Menstruationsstörungen bis hin zur Amenorrhoe (Korkia u. Stimson 1997), Klitorishypertrophie, und ein Tieferwerden der Stimme (Derman 1995). Zudem können temporärer Haarausfall (Glaser et al. 2012), aber auch ein übermäßiges Haarwachstum (Wollina et al. 2007) und ein Hirsutismus auftreten (Derman 1995).

\section{Nebenwirkungen bei Jugendlichen}

Bei Jugendlichen kann die Einnahme anaboler Steroide zu einer vorzeitigen Verknöcherung noch wachsender Knorpelstrukturen führen. Infolgedessen kommt es zu einem Stillstand des Längenwachstums und damit zu einer geringeren Körperendgröße (Dunkel 2011).

\section{Psychische Nebenwirkungen}

Bodybuilding im Zusammenhang mit der Einnahme anaboler Steroide kann die Aggressionsbereitschaft erhöhen (Yesalis et al. 1993). Zudem führt ein abruptes Absetzten des AAS nach längerem Missbrauch zu Kopfschmerzen, Schlaflosigkeit, Abgeschlagenheit sowie Stimmungsschwankungen bis hin zu einer erhöhten Suizidalität, da der Testosterongehalt reduziert ist (Medras u. Tworowska 2001; Trenton u. Currier 2005).

\section{Nebenwirkungen der Injektion von ölen}

Eine weitere Methode, die im Bereich des Bodybuilding häufig angewendet wird, um eine schnelle Vergrößerung des Muskels zu erreichen, ist das Injizieren von synthetischen (Sytol) oder teils natürlichen (steriles Sesamöl) Ölen direkt in entsprechende Muskelpartien (Ghandourah et al. 2012; Müller u. 
Liebau 2011). Dabei reichert sich das Öl im Muskel an und wird nur sehr langsam abgebaut, sodass es wie ein Implantat den Muskel optisch vergrößert. Nach mehrfachen Injektionen können starke Muskelschmerzen und -deformationen auftreten (Chandourah et al. 2012; Koopmann et al. 2005). Zudem zeigten sich allergische Reaktionen und Veränderungen der Haut, die bis hin zu Ölzysten und Fremdkörpergranulomen reichten (Schäfer et al. 2012; Banke et al. 2012, Ghandourah et al. 2012; Müller u. Liebau 2011). In einzelnen Fällen konnte eine irreversible Zerstörung des körpereigenen Muskels nachgewiesen werden (Banke et al. 2012). Auch eine Entzündung der Blutgefäße (Vakulitis) sowie eine Verhärtung des Bindegewebes (Fibrose) zeigte sich als Folge der ÖlInjektion (Koopman et al. 2005; Ghandourah et al. 2012). Im Blutbild konnte außerdem eine Erhöhung weißer Blutkörperchen nachgewiesen werden (Koopman et al. 2005).

\subsection{Fazit für den Umgang im klinischen Alltag}

Die Tatsache, dass ein großer Teil der im Breitensport und im Bodybuilding gebrauchten Muskelaufbaupräparate von Ärzten verschrieben wird, sollte zu einer kritischeren Verschreibungspraxis veranlassen. Aufgrund der weiten Verbreitung von anabolen-androgenen Steroiden und Stimulanzien im Breitensport und im Bodybuilding sollte in der Anamnese der Krankheitsgeschichte auch direkt nach einem Gebrauch dieser Substanzen gefragt werden, ebenso wie z.B. nach Alkohol und Nikotin gefragt wird. Besonders gilt dies natürlich, wenn es äußerlich sichtbare Hinweise wie stark ausgeprägte Muskeln oder Akne gibt. Auch affektive Störungen können bei Männern mit sehr hoher Muskelmasse ein Hinweis auf den vorangegangenen Konsum von anabolen Steroiden sein und sollten in der Anamnese beachtet werden.

Neben den somatischen Auswirkungen von Bodybuilding und dem Gebrauch von Muskelaufbaupräparaten sollten Ärzte und Psychotherapeuten auch die Motivation des Einzelnen für Bodybuilding erfragen. Der Hintergrund des Bestrebens, den Körper durch Bodybuilding umzuformen, können Körperbildoder Körperschemastörungen sein, die zu zwanghaften und suchtartigen Verhaltensweisen im Bereich des Trainings und der Ernährung führen können. Wenn der Betroffene andere soziale Aktivitäten in Familie, Beruf oder Freizeit reduziert oder aufgegeben hat, um trainieren oder einen Ernährungsplan einhalten zu können, ist das ein Hinweis auf eine vorliegende psychische Störung.

\section{Literatur}

Babusa B, Túry F (2012) Muscle dysmorphia in Hungarian non-competitive male bodybuilders. Eat Weight Disord 17, 49-53

Baghurst T (2012) Muscle dysmorphia and male body image: A personal account. Editorial Board, 125

Baghurst T, Hollander DB, Nardellac B, Haff GG (2006) Change in sociocultural ideal male physique: An examination of past and present action figures. Body Image 3, 87-91 
Baghurst T, Lirgg C (2009) Characteristics of muscle dysmorphia in male football, weight training, and competitive natural and non-natural bodybuilding samples. Body Image 6, 221-227

Bahrke MS, Yesalis CE (2004) Abuse of anabolic androgenic steroids and related substances in sport and exercise. Curr Opin Pharmacol 4, 614-620

Bain I (2007) The many faces of testosterone. Clinical Interventions in Aging 2, 567-576

Bak K, Cameron EA, Henderson IJP (2000) Rupture of the pectoralis major: a meta-analysis of 112 cases. Knee Surg Sports Traumatol Arthrosc 8, 113-9

Banke IJ, Prodinger PM, Waldt S, Weirich G, Holzapfel BM, Gardinger R, Rechl H (2012) Irreversible muscle damage in bodybuilding due to long-term intramuscular oil injection. Int I Sports Med 33, 829-834

Barlow IC, Benjamin BW, Brit P, Hughes CI (2002) Shoulder strength and range of motion characteristics in bodybuilders. I Strength Cond Res 16, 367-72

Baumeister RF (1986) Identity: Cultural change and the struggle for self. Oxford University Press New York

Berscheid E, Walster E, Bohrnstedt G (1973) The happy American body: A survey report. Psychology Today 7 , $119-131$

Beuker F, Gottschlak K, Schmidt HG, Tolkmitt U, Trepte H (1996) Sportartspezifische Verletzungen und Erkrankungen bei Gewichthebern. Med Sport 6, 133-135

Bhasin S (2000) The dose-dependent effects of testosterone on sexual function and on muscle mass and function. Mayo Clin Proc. Mayo Suppl S70-75; discussion S75-6.

Bhasin S, Woodhouse L, Casaburi R, Singh AB, Bhasin D, Berman N, Chen X, Yarasheski KE, Magliano L, Dzekov C, Dzekov I, Bross R, Phillips I, Sinha-Hikim I, Shen R, Storer TW (2001) Testosterone dose-response relationships in healthy young men. Am / Physiol Endocrinol Metab 281, E1172-1181

Birkeland KI, Jorgensen J, Hemmersbach P (1994) Endocrine effects of doping with androgenic anabolic steroids. Tidsskr Nor Laegeforen 114, 426-428

Boos C, Wulff P (2001) Der Medikamentenmissbrauch bei Freizeitsportlern im Fitnessbereich. Öffentliche Anhörung zum Doping im Freizeit- und Fitnessbereich. Protokoll der 38. Sitzung des Sportausschusses. 14. Wahlperiode, S. 115-152

Boos C, Wulff P, Kujath P, Bruch HP (1998) Medikamentenmissbrauch beim Freizeitsportler im Fitnessbereich. Deutsches Ärzteblatt 95, C-708-C-712

Breitenstein B (2003) Natural Bodybuilder: Eine Erfolgsstory ohne Doping. BoD-Books on Demand

Breitenstein B (2007) Power-Bodybuilding Erfolgreich, Natürlich, Gesund. Rowohlt Taschenbuch Verlag

Brown EW, Kimball RG (1983) Medical history associated with adolescent powerlifting. Pediatrics 72, 636-644

Brownell KD (1991) Personal responsibility and control over our bodies: When expectation exceeds reality. Health Psychology 10, 303-310

Bundesinstitut für Sportwissenschaft (2001) Ergebnisse der A-Analysen von Bodybuildingwettkämpfen. Zusammengestellt aus den Dopingpressekonferenzen 1996 bis 2000, BISp Bonn

Bundesrat: Drucksache 223/07 (2007). Gesetzentwurf der Bundesregierung. Entwurf eines Gesetzes zur Verbesserung der Bekämpfung des Dopings im Sport: 30.3.2007

Cabasso A (1994) Peliosis hepatis in a young adult bodybuilder. Med Sci Sports Exerc 26, 2-4

Cafri G, Olivardia R, Thompson JK (2008) Symptom characteristics and psychiatric comorbidity among males with muscle dysmorphia. Compr Psychiatry 49, 374-379

Cash TF (2004) Body image: Past, present, future. Body Image 1, 1-5

Cash TF, Morrow JA, Hrabosky II, Perry AA (2004) How has body image changed? A cross-sectional investigation of college women and men from 1983 to 2001. I Consult Clin Psych 72, 1081-1089

Cash TF, Winstead BW, Janda LH (1986) The great American shape up: Body image survey report. Psychology Today 20, 30-37

Clasing D (2004) Dopingmittel. In: Clasing D. (Hrsg.), Doping und seine Wirkstoffe. 53-63. Spitta Verlag Balingen

Daniel S, Bridges SK (2010) The drive for muscularity in men: Media influences and objectification theory. Body Image 7, 32-38

De Souza GL, Hallak I (2011) Anabolic steroids and male infertility: A comprehensive review. BJU Int. 108, 1860-1865 
Deloitte/DSSV-Studie (2010) Der Deutsche Fitnessmarkt 2010. Online unter: www.deloitte.com/view/de_DE/ de/presse/pressemitteilungen/8ef814051900f210VgnVCM2000001b56f00aRCRD.htm (abgerufen am 02.10.2013)

Deloitte/DSSV-Studie (2011) Der Deutsche Fitnessmarkt 2011. Online unter: www.deloitte.com/view/de_DE/ de/branchen/consumerbusinessandtransportation/tourism-hospitality-leisure/leisure/health-fitness/e7 0236952b6f7210VgnVCM200000bb42f00aRCRD.htm (abgerufen am 02.10.2013)

Derman RJ (1995) Effects of sex steroids on women's health: Implications for practitioners. Am I Med 98, 137-143

Dickerman RD, McConathy WJ, Zachariah NY (1997) Testosterone, sex hormone-binding globulin, lipoproteins, and vascular disease risk. / Cardiovasc Risk 4, 363-366

Donati S (2007) World traffic in doping substances. Montreal. Online unter: www.wada-ama.org/rtecontent/ document/Donati_Report_Trafficking_2007-03_06.pdf (abgerufen am 02.10.2013)

DSSV e.V. (2008) Kundenzufriedenheit in Fitnessanlagen. Inno fact 2008. Online unter: www.dssv.de/filead$\mathrm{min} /$ kunden/Presseportal/Innofact/innofact.pdf (abgerufen am 02.10.2013)

Dunkel L (2011) Treatment of idiopathic short stature: Effects of gonadotropin-releasing hormone analogs, aromatase inhibitors and anabolic steroids. Horm Res Paediatr 76 Suppl 3, 27-29

EMNID-Studie Fit-/Wellnessmarkt (2002/2003) Verband Deutscher Fitness- und Freizeitunternehmer (VDF), BODYLIVE Fachverlage GmbH \& Co KG Karlsruhe

Frankle MA, Eichberg R, Zachariah SB (1988) Anabolic androgenic steroids and a stroke in an athlete: Case report. Arch Phys Med Rehabil 69, 632-633

Froehner M, Fischer R, Leike S, Hakenberg OW, Noack B, Wirth MP (1999) Intratesticular leiomyosarcoma in a young man after high dose doping with Oral-Turinabol: A case report. Cancer 86, 1571-1575

Fussell SW (1991) Muscle: Confessions of an unlikely bodybuilder. New York: Avon Books

Garner DM (1997) The body image survey results. Psychology Today 30, 30-44

Ghandourah S, Hofer M), Kießling A, El-Zayat B, Schofer MD (2012) Painful muscle fibrosis following synthol injections in a bodybuilder. J Med Case Reports 6, 248

Gesetz vom 2.3.1994 zu einem Übereinkommen vom 16.9.1989 gegen Doping, BGBL. 1994 II S. 334

Giraldi W (2011) Freaky Beasts: Revelations of a one-time bodybuilder. Online unter: williamgiraldi.com/wpcontent/uploads/2011/08/freaky.beasts1.pdf (abgerufen am 02.08.2013)

Glaser RL, Dimitrakakis C, Messenger AG (2012) Improvement in scalp hair growth in androgen-deficient women treated with testosterone: A questionnaire study. Br | Dermatol 166, 274-278

Goertzen M, Schöppe K, Lange G, Schulitz KP (1989) Verletzungen und Überlastungsschäden beim Bodybuilding und Powerlifting. Sportverl Sportschad. 3, 323-6

Goldfield GS, Blouin AG, Woodside DB (2006) Body image, binge eating, and bulimia nervosa in male bodybuilders. Can | Psychiatry 51, 160-168

Granhead H, Morelli B (1988) Low back pain among retired wrestlers and heavyweight lifters. AM I Sprts Med 16, 530-533

Haas U (2004) Rechtliche und organisatorische Grundlagen der Dopingbekämpfung in Deutschland. In: Clasing D (Hrsg.) Doping und seine Wirkstoffe.166-186. Spitta Verlag Balingen

Habscheid W, Abele U, Dahm HH (1999) Severe cholestasis with kidney failure from anabolic steroids in a bodybuilder. Dtsch Med Wochenschr 124, 1029-1032

Hartgens F, Rietjens G, Keizer HA, Kuipers H, Wolffenbuttel BHR (2004) Effects of androgenic-anabolic steroids on apolipoproteins and lipoprotein. Br I Sports Med 38, 253-259

Hartung R, Gerth I, Fünfstück R, Gröne HJ, Stein G (2001) End-stage renal disease in a bodybuilder: A multifactorial process or simply doping? Nephrol Dial Transplant 16, 163-165

Heatherton TF, Nichols P, Mahamedi F, Keel P (1995) Body weight, dieting, and eating disorder symptoms among college students 1982 to 1992. Am J Psychiat 152, 1623-1629

Hurley BF, Seals DR, Hagberg JM, Goldberg AC, Ostrove SM, Holloszy J0, Wiest WG, Goldberg AP (1984) Highdensity-lipoprotein cholesterol in bodybuilders $v$ powerlifters. Negative effects of androgen use. JAMA $252,507-513$ 
Hurst R, Hale B, Smith D, Collins D (2000) Exercise dependence, social physique anxiety, and social support in experienced and inexperienced bodybuilders and weightlifters. British Journal of Sports Medicine 34, $431-435$

Jefferson T (1998) Muscle, "Hard men" and "Iron" Mike Tyson: Reflections on desire, anxiety and the embodiment of masculinity. Body \& Society 4, 77-98

Jerosch J, Ritchen A, Marquart M (1989) Sonographische Befunde am Schultergelenk von Bodybuildern. Dtsch Z Sportmed 40, 437-42

Johnson CA (2000) Use of androgens in patients with renal failure. Semin Dial 13, 36-39

Kau D (2013) Vorsitzender des National Athletic Commitee Germany (NAC), persönliche Mitteilung am 25.01.2013

Kister L (2007) Anabolika-Forscher Kistler: „Irgendwann macht es halt bumm.“ Online unter: www.spiegel.de/ sport/sonst/anabolika-forscher-kistler-irgendwann-macht-es-halt-bumm-a-459177.html (abgerufen am 02.10.2013)

Klein AM (2007) Special issue. Sport in Society, 1073-1119

Klein W, Schulitz KP, Neumann C (1979) Orthopädische Probleme beim Bodybuilding. Dtsch Z Sportmed 30, 296-306

Kochakian CD (1993) History chemistry and pharmacodynamics of anabolic-androgenic steroids. Wien Med Wochenschr 143, 359-363

Koopman M, Richter C,Parren RIM, Janssen M (2005) Bodybuilding, sesame oil and vasculitis. Oxford Journals, Medicine, Rheumatology 44, pp 1135

Korkia P, Stimson GV (1997) Indications of prevalence, practice and effects of anabolic steroid use in Great Britain. Int | Sports Med 18, 557-562

Kraus SL, Emmert S, Schön MP, Haenssle HA (2012) The dark side of beauty: Acne fulminans induced by anabolic steroids in a male bodybuilder. Arch Dermatol 148, 1210-1212

Law C, Labre MP (2002) Cultural standards of attractiveness: A thirty-year look at changes in male images in magazines. Journalism \& Mass Communication Quarterly 79, 697-711

Li P (2004) The theoretical construct and application of "sub-sport event." Journal of Tianjin Institute of Physical Education 19, 68-70

Lin CA (1998) Uses of sex appeals in prime-time television commercials. Sex Roles 38, 461-475

LL Cool I (2011) Be your best self. Men's Fitness, March 1, 2011

Leit RA, Pope HG, Gray II (2001) Cultural expectations of muscularity in men: The evolution of Playgirl centerfolds. International Journal of Eating Disorders 29, 90-93

Lisiewicz |, Fijałkowski P, Sankowski I (1999) Ischemic cerebral stroke and anabolic steroids (case report). Neurol Neurochir Pol 32, 137-139

Lippert HD (1999) Achtes Gesetz zur Änderung des Arzneimittelgesetzes. NJW 52, 837-838

Martorana G, Concetti S, Manferrari F, Creti S (1999) Anabolic steroid abuse and renal cell carcinoma. J Urol 62, 2089

Mayerhausen W, Riebel B (1989) Acne fulminans following use of anabolic steroids. Z Hautkr 64, 879-880

McCarthy K, Tang AT, Dalrymple-Hay MJ, Haw MP (2000) Ventricular thrombosis and systemic embolism in bodybuilders: Etiology and management. Ann Thorac Surg 70, 658-660

McCreary DR, Hildebrandt T B, Heinberg LI, Boroughs M, Thompson JK (2007) A review of body image influences on men's fitness goals and supplement use. American Journal of Men's Health 1, 307-316

McKillop G, Todd IC, Ballantyne D (1986) Increased left ventricular mass in a bodybuilder using anabolic steroids. Brit / Sports Med 20, 151-152

Medraś M, Tworowska U (2001) Treatment strategies of withdrawal from long-term use of anabolic-androgenic steroids. Pol Merkur Lekarski 11, 535-538

Melnik B, Jansen T, Grabbe S (2007) Abuse of anabolic-androgenic steroids and bodybuilding acne: an underestimated health problem. I Dtsch Dermatol Ges 5, 110-117

Melnik BC (2009) Androgen abuse in the community. Curr Opin Endocrinol Diabetes Obes. 16:218-223

Müller A (2011) Bodybuilding und Kraftsport in der DDR. Sportverlag Strauß

Müller N, Liebau I (2011) Komplikationen durch Injektion von synthetischen und natürlichen Ölen zur optischen Muskelvergrößerung bei Bodybuildern. Düsseldorf German Medical Science GMS Publishing House 
Mosley PE (2009) Bigorexia: Bodybuilding and muscle dysmorphia. European Eating Disorders Review 17, 191-198

Murray SB, Rieger E, Touyz SW, De la Garza García L (2010).Muscle dysmorphia and the DSM-V conundrum: Where does it belong? A review paper. International Journal of Eating Disorders 43, 483-491

Nakao A, Sakagami K, Nakata Y, Komazawa K, Amimoto T, Nakashima K, Isozaki H, Takakura N, Tanaka N (2000) Multiple hepatic adenomas caused by long-term administration of androgenic steroids for aplastic anemia in association with familial adenomatous polyposis. I Gastroenterol 35, 557-562

Neild D (1995) Gynaecomastia in bodybuilders. Br I Clin Pract 49, 172

Martin NM, Abu Dayyeh BK, Chung RT (2008) Anabolic steroid abuse causing recurrent hepatic adenomas and haemorrhage. World I Gastroenterol 14, 4573-4575

Nieminen MS, Rämö MP, Viitasalo M, Heikkilä P, Karjalainen I, Mäntysaari M, Heikkilä J (1996) Serious cardiovascular side effects of large doses of anabolic steroids in weight lifters. Eur Heart I 17, 1576-1583

Olivardia R, Pope HG, Borowiecki II, Cohane GH (2004) Biceps and body image:The relationship between muscularity and self-esteem, depression, and eating disorder symptoms. Psychology of Men \& Masculinity 5 , $112-120$

Parish T, Baghurst T, Turner R (2010) Becoming competitive amateur bodybuilders: Identification of contributors. Psychology of Men \& Masculinity 11, 152-159

Parkinson AB, Evans NA (2006) Anabolic androgenic steroids: A survey of 500 users. Med Sci Sports Exerc 38, $644-651$

Payne IR, Kotwinski PI, Montgomery HE (2004) Cardiac effects of anabolic steroids Heart 90, 473-475

Pickett TC, Lewis RI, Cash TF (2005) Men, muscles, and body image: Comparisons of competitive bodybuilders, weight trainers, and athletically active controls. British Journal of Sports Medicine 39, 217-222

Pöhlmann K, Joraschky P (2006) Körperbild und Körperbildstörungen: Der Körper als gestaltbare Identitätskomponente. Psychother Dialog 7, 191-195

Pope H, Brower K (2005) Anabolic-androgenic steroid abuse. In: Sadock B, Sadock V (Eds.), Comprehensive textbook of psychiatry. 1318-1328 Philadelphia Lippincott Williams \& Wilkins

Pope HG, Gruber A], Choi P, Olivardia R, Phillips KA (1997) Muscle dysmorphia. An under-recognised form of body dysmorphia disorder. Psychosomatics 38, 548-557

Pope HG, Katz DL, Hudson II (1993) Anorexia nervosa and "reverse anorexia" among 108 male bodybuilders. Comprehensive Psychiatry 34, 406-409

Pope HG, Katz DL (1994) Psychiatric and medical effects of anabolic androgenic steroid use: A controlled study of 160 athletes. Arch Gen Psychiatry 51, 375-382

Pope HG, Phillips KA, Olivardia R (2000) The Adonis complex: The secret crisis of male body obsession. New York Free Press

Révai T, Sápi Z, Benedek S, Kovács A, Kaszás I, Virányi M, Winkler G (2003) Severe nephrotic syndrome in a young man taking anabolic steroid and creatine long term. Orv Hetil 144, 2425-2427

Ritsch M (2005) Bodybuilding. In: Grifka I (Hrsg.) Praxiswissen Halte- und Bewegungsorgane. 145-151. Thieme

Rüdiger C, Parzeller M (2007) Dopingbekämpfung im Sport in Deutschland de lege ferenda im Kontext europäischer Normgebung. Sportorthopädie Sporttraumatologie 23

Ryan A) (1981) Anabolic steroids are fool's gold. Fed Proc 12, 2682-2688

Sandow $\mathrm{E}$ (1905) Bodybuilding or man in the making - how to become healthy and strong. London

Sachtleben TR, Berg KE, Elias BA, Cheatham IP, Felix GL, Hofschire PI (1993) The effects of anabolic steroids on myocardial structure and cardiovascular fitness. Med Sci Sports Exerc 25, 1240-1245

Schäfer CN, Hvolris |, Plambech M (2011) Muscle enhancement using intramuscular injections of oil in bodybuilding: Review on epidemiology, complications, clinical evaluation and treatment. Eur Surg 44, 109-115

Schänzer W, Thevis M (2007) Doping im Sport. Medizinische Klinik Journal 102, 631-646

Scheller I (2010). No Sports! Zur Ästhetik des Bodybuildings. Stuttgart Franz Steiner Verlag

Scheller I (2012) Arnold Schwarzenegger oder Die Kunst ein Leben zu stemmen. Stuttgart Franz Steiner Verlag Schilder P (1923) Das Körperschema: Ein Beitrag zur Lehre vom Bewusstsein des eigenen Körpers. Julius Springer Schwarzenegger A (1986). Das große Bodybuilding Buch. München Wilhelm Heyne Verlag. 
Scott MI Jr, Scott M) 3rd, Scott AM (1994) Linear keloids resulting from abuse of anabolic androgenic steroid drugs. Cutis 53, 41-3

Sheffield-Moore M, Urban RJ (2004) An overview of the endocrinology of skeletal muscle. Trends Endocrinol Metabol 15,110-115

Shepsis AA, Grafe MW, Jones HP, Lemos MJ (2000) Rupture of the pectoralis major muscle. AM I Sports Med 28, 9-15

Smith ACT, Stewart B (2012) Body perceptions and health behaviors in an online bodybuilding community. Qualitative Health Research 22, 971-985

Spitz L (1989) Fit mit Bodybuilding. Niederhausen Falken Bücherei

Steinbrück K (1999) Epidemiologie von Sportverletzungen - 25 lahre-Analyse einer sportorthopädisch-traumatologischen Ambulanz. Sportverl Sportschad 13, 38-52

Stockert B von (1986) Frauenbodybuilding: Die Lust an der Verführung oder das Begehren des Ideals. Arbeiten aus dem Institut für Sportwissenschaften der FU Berlin-Sozialwissenschaftliche Reihe-Band 7. Köln Verlag Buch \& Sport

Sullivan ML, Martinez CM, Gennis P, Gallagher El (1998) The cardiac toxicity of anabolic steroids. Prog Cardiovasc Dis $41,1-15$

Stimac D, Milić S, Dintinjana RD, Kovac D, Ristić S (2002) Androgenic/Anabolic steroid-induced toxic hepatitis. I Clin Gastroenterol 35, 350-352

Torres-Calleja |, González-Unzaga M, DeCelis-Carrillo R, Calzada-Sánchez L, Pedrón N (2001) Effect of androgenic anabolic steroids on sperm quality and serum hormone levels in adult male bodybuilders. Life Sci 68, $1769-74$

Torres-Calleja I, De Celis R, Gonzalez-Unzaga M, Pedrón-Nuevo N, Fertil Steril (2000) Effect of androgenic anabolic steroids on semen parameters and hormone levels in bodybuilders 74, 1055-6

Trenton Al, Currier GW (2005) Behavioural manifestations of anabolic steroid use. CNS Drugs 19, 571-95

Vanberg P, Atar D (2010) Androgenic anabolic steroid abuse and the cardiovascular system. Handb Exp Pharmacol 195, 411-57

Velayudham LS, Farrell GC (2003) Drug-induced cholestasis. Expert Opin Drug Saf 2, 287-304

Vogt I (2007) Männer und Medikamente: Zum Beispiel Dopingmittel. Men and Pharmaceuticals: For Example Doping. Suchttherapie 8, 103-107

Wedemeyer B (1999) Der Athletenvater Theodor Siebert (1866-1961): Eine Biographie zwischen Körperkultur, Lebensform und Esoterik. Göttingen Nobert Klatt Verlag

Wedemeyer-Klowe B (2004) „Der neue Mensch“: Körperkultur im Kaiserreich und in der Weimarer Republik. Würzburg Könighausen \& Neumann GmbH

Weinmann W (2004) Das Kampfsportlexikon von Aikido bis Zen. Verlag Weimann Berlin

Wikipedia (2013) Bodybuilding. Online unter: http://de.wikipedia.org/wiki/Bodybuilding (abgerufen am 02.10.2013)

Wollina U, Pabst F, Schönlebe I, Abdel-Naser MB, Konrad H, Gruner M, Haroske G, Klemm E, Schreiber G (2007) Side-effects of topical androgenic and anabolic substances and steroids. A short review. Acta Dermatoven APA 16(3)

World Anti-Doping Agency (WADA) (2013) The 2013 prohibited list. Online unter: http://www.wada-ama.org/ Documents/World_Anti-Doping_Program/WADP-Prohibited-list/2013/WADA-Prohibited-List-2013-EN.pdf (abgerufen am 02.10.2013)

Würzberg G (1987) In den Maschinenhallen der neuen Körperkultur. Reinbek bei Hamburg: Rowohlt Taschenbuchverlag

Yeater R, Reed C, Ullrich I, Morise A, Borsch M (1996) Resistance trained athletes using or not using anabolic steroids compared to runners: effects on cardiorespiratory variables, body composition, and plasma lipids. Br I Sports Med 30, 11-14

Yesalis CE, Kennedy N], Kopstein AN, Bahrke MS (1993) Anabolic-androgenic steroid use in the United States. JAMA 270, 1217-21

Zhang Y, Zhou W, Song G, Li J, Yuan H (2002) Sociological consideration of health promotion by exercises in middle-aged people. Journal of Beijing University of Physical Education 25, 436-438 